\title{
Administración y Estado en el contexto post Covid-19: ¿Hacia un nuevo tipo de vínculo?
}

\section{Administration and State in the post Covid-19 context: Towards a new type of bond?}

\author{
Josep Pont Vidal \\ Universidade Federal do Pará - UFPA (Brasil) \\ ORCID: https://orcid.org/0000-0002-6214-6895 \\ joseppontvidal@gmail.com
}

\begin{abstract}
NOTA BIOGRÁFICA
Licenciado en Sociología política (Universitat Bielefeld, Alemania), Master en Sociología (Bielefeld), doctorado en Sociología Política (Universidad de Barcelona, 1997). Profesor titular e investigador del Núcleo de Altos Estudios Amazónicos (NAEA-UFPA). Experiencia como profesor visitante en varias universidades Latino-americanas (Nicaragua, Colombia, el Colegio de Jalisco, México), brasileiras (UECE; UFBA; PUC-SP; UNESP; UFES, Fundação Getúlio Vargas) y europeas. Experiencia y actuación en el área de Políticas Públicas y Gobernanza con trabajos en la Administración Publica desarrollados en el Banco Interamericano de Desarrollo (BID - Washington), Universidad Autónoma de Barcelona (UAB), Instituto de Desenvolvimento Econômico, Social e Ambiental do Pará (IDESP), y Instituto de Pesquisas Aplicadas (IPEA) (Brasilia). Actuación en las áreas de Teoría de las organizaciones, Administración y Gestión pública, Políticas Públicas. Coordinador del Programa de Pos-graduación del Master en Gestión Pública para el Desarrollo (2012-2014).
\end{abstract}

\section{RESUMEN}

La pandemia de Covid-19 ha tenido profundos impactos en todas las sociedades. El Estado y la Administración no han quedado al margen sino que se encuentran en un entorno caracterizado por cambios dinámicos, desconocidos y contingentes. Las transformaciones iniciadas unos años antes sobre la función y extensión del Estado se han acentuado, y las administraciones tendrán que adaptarse a unas condiciones muy inestables: cambios en la economía y de los actores políticos, reformulación rápida de métodos, nuevas demandas, reformulación de la relación con la sociedad. En este escenario aparecen dos cuestiones centrales. Una primera de carácter descriptivo, sobre la transformación del Estado y la administración en un entorno inseguro, complejo y contingente. En la segunda de índole metodológicoconceptual, aparece la necesidad de procurar y aplicar conceptos y lógicas con capacidad del diálogo interdisciplinario y con la suficiente idoneidad y sofisticación para describir esta complejidad.

\section{PALABRAS CLAVE}

Administración pública; Covid-19; contingencia; riesgo; Estado.

\begin{abstract}
The Covid-19 pandemic has had profound impacts on all societies. The State and the Administration have not been left out but are in an environment characterized by dynamic changes and contingent. The changes initiated a few years before on the function and extension of the State have been accentuated, and the administrations will have to adapt to very unstable conditions: changes in the economy and political actors, rapid reformulation of methods, new demands, relationship with the society. Two central questions appear
\end{abstract}


GAPP. Nueva Época - N. ${ }^{\circ} 26$, julio 2021 - ISSN: 1989-8991 - DOI: https://doi.org/10.24965/gapp.i26.10798 - [Págs. 33-47]

Administración y Estado en el contexto post Covid-19: ¿Hacia un nuevo tipo de vínculo?

Josep Pont Vidal

in this scenario. The first one is descriptive in nature, about the transformation of the State and the Public Administration in an insecure, complex and contingent environment. In the second of a methodologicalconceptual nature, there is a need to seek and apply concepts and logics capable of interdisciplinary dialogue and with sufficient suitability and sophistication to describe this complexity.

\title{
KEYWORDS
}

Public administration; Covid-19; contingency; risk; State.

\begin{abstract}
SUMARIO
INTRODUCCIÓN Y PROBLEMATIZACIÓN. 1. HACIA OTRA LÓGICA METODOLÓGICA Y CONCEPTUAL. 1.1. SOCIEDADES COMPLEJAS COMO PERSPECTIVA DE PARTIDA. 1.2. COMPLEJIDAD, CONTINGENCIA Y RIESGO. 1.3. HIPERCOMPLEJIDAD Y ADMINISTRACIÓN. 1.4. CONTINGENCIA, RIESGO Y OBSERVACIÓN. 2. ADMINISTRACIÓN PÚBLICA. 2.1. LOS GÉNESIS DE LOS ANÁLISIS ACTUALES. 2.2. ¿DÓNDE NOS ENCONTRAMOS HOY? 2.3. ESCENARIO POST COVID-19: MODELOS AUTO-EXCLUYENTES. 2.4. ESTADO REDUCIDO JERÁRQUICO. 2.5. ESTADO EXTENSIVO: «ESTADO-CÉNTRICO». CONCLUSIONES Y UNAS REFLEXIONES.
\end{abstract}

\section{INTRODUCCIÓN Y PROBLEMATIZACIÓN}

La pandemia del Covid-19 ha sido la causa de que el conjunto de gobiernos, administraciones e instituciones públicas hayan tomado una serie de medidas drásticas de control, vigilancia, fiscalización y de sanciones, antes no conocidas en las democracias liberales occidentales. Estas medidas han incidido y afectado profundamente no solo la vida cotidiana, el trabajo, el tiempo libre, la economía, el deporte, sino que también han acelerado procesos ya iniciados unos años antes e iniciado otros de reformulación de las dinámicas de la administración, sobre las responsabilidades y funciones del Estado, en su tipología y estructura de gobernanza regulatoria y las políticas públicas regulatorias. A esta nueva situación dinámica y contingente, hay que añadir las medidas que los gobiernos de todo el mundo han tomado referentes a las restricciones de la libertad constitucional: confinamiento de la población, restricción de movimientos, vigilancia y control masivos por medio de las posibilidades de big-data, y de medidas coercitivas. Recientes publicaciones y descubrimientos virológicos ya anticipan un escenario pesimista para los próximos años en el que la «nueva normalidad» será convivir con nuevos virus, en el contexto de posible reaparición del coronavirus, en suma, un escenario de incertezas.

En esta situación, la administración pública como un elemento central de la estadidad, asume paulatinamente funciones y competencias del poder ejecutivo y, como tal, ha sido responsable de la implementación de las normas especiales, la concesión y administración de servicios, la realización de tareas regulatorias con intervenciones y permisos, la expansión y mantenimiento de la infraestructura, entre otras atribuciones y responsabilidades. La situación excepcional de contingencia creada por la pandemia del coronavirus, ha acelerado que los valores de referencia, atribuciones y competencias del Estado y la administración estén cambiando de manera acelerada y se está cuestionando la manera como han funcionado hasta ahora. El análisis de esta dinámica esta siendo abordada en diversos workshops y conferences digitales internacionales recientes ${ }^{1}$. En ellos aparecen nuevas temáticas (migraciones, turismo de masas, ecosistemas, metodologías) en los que se hace evidente la interdisciplinariedad y en los que se ha destacado la necesidad de aplicar nuevos conceptos en la administración para el análisis de la situación de contingencia y pos-pandemia actual, aunque la observación y aplicación efectiva de estos cambios todavía chocan en la práctica y en la academia.

Plantear un análisis de la relación formada por el Estado y la administración en este contexto excepcional, requiere unas observaciones y supuestos previos que es preciso aclarar. En primer lugar, es necesario iniciar un diálogo entre las áreas de conocimiento que de manera habitual han abordado estos ámbitos, y que son la ciencia política, la administración y la sociología. Inevitablemente requiere iniciar un diálogo entre ellas, desde la concepción de la interdisciplinaridad. La voluntad del diálogo interdisciplinario por sí solo no

Zukunftskongress-digital. Staat und Verwaltng. Die Leitveranstalgung des Public Sectors fur Digitalen Wandel, Además, Berlin el 16 de junio de 2020, ICPAD (Paris), IASIA (South Africa), ICPAPSP (Londres), ICPPAE (Barcelona, Londres), ICGPARI (Berlín, Roma), Anpad, EnAnpad (Brasil), CLAD (Lisboa), entre otros. 
es suficiente. Ha sido habitual que éste se limitara a una mera colocación simultánea y paralela de disciplinas y discursos, dando origen más bien una codisciplinariedad, sin un avance sustancial conceptual en la descripción de los fenómenos sociales o de naturaleza político-administrativa. En segundo lugar, consideramos que la idea de asumir la descripción de los procesos de toma de decisiones, transformación del Estado y la administración, y su nexo con la sociedad, es un proceso de alta complejidad dado el actual contexto. Requiere de la aplicación unos supuestos en el ámbito conceptual y metodológico con capacidad observacional de identificar y describir los vínculos emergentes en su totalidad. Proponemos aquí iniciar el análisis a partir de la noción de complejidad. Esta predisposición, como se ha manifestado en las conferencias, ha entrado lentamente en la mente de los profesionales y académicos en las citadas disciplinas, aunque los conceptos subyacentes de la complejidad todavía no han normalizado para la observación y análisis en estos ámbitos.

El escenario creado por el Covid-19 -como otros recientes fenómenos, asociados a riesgos y peligros de naturaleza ontológica- (rotura de presas, ensayos de biotecnología, nanotecnologías, etc.) ha mostrado la hipercomplejidad de las sociedades actuales, al vincular todos los ámbitos de nuestra vida, y en consecuencia todas las disciplinas y áreas de conocimiento. Por otro lado, se han puesto de manifiesto los límites del análisis de la lógica observacional del tradicional modelo explicativo causa-efecto, o variable independiente y variable dependiente. Sobresalen dos preguntas centrales, que vinculan los planos descriptivo, metodológico-conceptual y analítico. La primera de carácter metodológico y conceptual es: ¿De qué conceptos y metodologías disponemos que sobrepasen las limitaciones de las metodologías habituales de las ciencias de la administración, sociología y política para observar y describir esta nueva complejidad que se nos presenta? La segunda, de carácter descriptivo se dirige a la pregunta: ¿Cómo se manifiesta este nuevo entorno creado la recomposición entre el Estado y la administración?

Responder previamente estas preguntas a partir de los conceptos comprendidos en las corrientes teóricas tradicionales (neo-marxismo, institucionalismo) trae el riego de analizar el fenómeno a partir de respuestas repetidas y por lo tanto ineficaces. Estas han mostrado los límites de la explicación de la racionalidad humana que postulan las corrientes neo-institucionalistas, como tampoco el análisis del «poder» ha podido explicar cómo se produce la acción en un sistema social o personal. Así aparece necesario la procura y profundización de instrumentos conceptuales y metodologías con capacidad para describir lo más exacto posible las sociedades en este periodo actual. La hipótesis sustantiva es que la irrupción de la pandemia ha provocado cambios que afectan también al vínculo entre el Estado la administración, en particular en sus competencias y modo de gobernar, y en la delimitación, autonomía, extensión y orientación de la administración.

El escrito se estructura en dos apartados. En el primero abordamos la lógica metodológica circular dinámica y los conceptos de contingencia y riesgo, como conceptos vinculados con la complejidad de las sociedades actuales, cuya aplicación en la administración pública ha sido escasamente analizada. En el segundo, por medio del marco de presentación de la hipercomplejidad, describimos los cambios y las tendencias que se están produciendo en las formas de actuación y del papel del Estado y de la administración sus impactos en las sociedades.

\section{HACIA OTRA LÓGICA METODOLÓGICA Y CONCEPTUAL}

Estado y administración ya han sido analizados en numerosas publicaciones desde perspectivas científicas diferentes, y ha sido habitual, describir ambos ámbitos o esferas como un único, en que referirse al Estado implica de manera automática su administración o administraciones. Esta perspectiva ha sido abordada en la óptica del neo-marxismo a parir de ámbitos sociales diferenciados (Jessop, 2016), en la neoinstitucional en sus variantes politológica y administrativa (Lenk, 2014).

La óptica constructivista-sistémica presupone la separación del Estado y la Administración como dos esferas o ámbitos diferenciados, poseyendo cado uno de ellos una autonomía y capacidad de acción independiente, aunque vinculados directamente con la sociedad (Luhmann, 2010; Willke, 1987; 1993; 1999). En esta perspectiva Estado y administración son dos ámbitos diferenciados, y por lo tanto autónomos, al poseer cada uno de ellos una dinámica diferente y la capacidad de crear sus propias estructuras mediante operaciones internas. Al tratarse de ámbitos diferentes, posibilita presentarlos cada uno de ellos con un entorno propio, con el que interactúa por medio de comunicaciones. Estas pueden ser recibidas (aceptadas, ignoradas o rechazadas) y son devueltas a su entorno por medio de nuevas comunicaciones. Este proceso se presenta por medio de una circularidad en la que interviene el sistema y su entorno. Su análisis epistemológico y filosófico ya ha sido objeto de diversas publicaciones. 
El abordaje metodológico que proponemos para el análisis de los cambios hipotéticos y visibles que ya están teniendo lugar en la relación entre la administración y el Estado, se sustentan en tres supuestos metodológicos centrales, los cuales permiten una descripción precisa sobre las dinámicas internas y externas entre estos dos ámbitos: la lógica observacional circular, la presentación de la complejidad, y la propuesta de diálogo interdisciplinario. Exponemos la relación entre ellos y sus posibilidades metodológicas para la observación. Sopesamos también que este análisis requiere de una explicación conceptual más detallada y separada, en la que es necesario describir y analizar el vínculo existente entre la lógica observacional para la obtención de conocimiento, los conceptos que utilizaremos y su semántica y significado, y el tipo de observación que se propone para este caso, puesto que no es posible separar o reducir a compartimentos estancos estos tres niveles que conforman una observación.

La lógica observacional habitual de obtención del conocimiento se basa en el binomio causa y efecto. Si bien este diseño ha contribuido para la obtención del conocimiento en el ámbito de la sociología y de la ciencia política, también ha mostrado sus límites, ya que un fenómeno tiene múltiples causas con múltiples efectos, que a su vez, son productores de nuevas causas. Este hecho se ha puesto de manifiesto en el análisis y descripción de la reciente pandemia del coronavirus: la causa de la rapidez en la extensión de la pandemia ¿se ha debido a la venta de animales salvajes en el mercado de Wuhan en China?, o por el contrario, ¿en una secuencia de causas derivadas de unas leyes permisivas adoptadas anteriormente por el gobierno de este país para este tipo de comercio?, ¿o resulta de la tomada de decisiones jerárquicas y burocráticas previas y de la opacidad del Gobierno, y a la falta de transparencia del partido en el poder en China?. Siguiendo este racionamiento, incluso se podría cuestionar el modelo de crecimiento rápido y la destrucción de los espacios naturales y de la fauna en este país. En las sociedades occidentales, en la expansión de la pandemia surgen del mismo modo preguntas sobre las causas iniciales ¿Qué medidas fueron asumidas para identificar la pandemia cuando estaba en sus inicios? ¿Se ha debido a la lentitud en reconocer su gravedad y en tomar medidas de aislamiento y confinamiento necesarias, o a las estrategias equivocadas para frenar o combatir la pandemia tomadas por los gobiernos occidentales?, ¿El confinamiento total de la población fue más efectivo que el disponer de un sistema sanitario que permitiera realizar los tests selectivos ultrasensibles de PCR de manera masiva, previos entre la población?, o incluso, ¿Debates políticos instrumentalizados favorecieron su extensión y mala gestión? Aparecen pues numerosas causas, con diversos y dramáticos efectos.

Su transposición al análisis de la relación Estado-administración no puede pues reducirse al análisis del tipo de comercio en un mercado, a una decisión jurídica previa, o a una única responsabilidad del Estado y de la gobernanza regulatoria o del Gobierno, puesto que, a nuestro entender, no soluciona el problema de la complejidad que emerge en este fenómeno como tampoco ofrece la posibilidad de una identificación clara y la descripción y análisis de las múltiples variables que intervienen y sus intersecciones o comunicaciones e influencias mutuas. Ante la complejidad del fenómeno y de las múltiples variables que intervienen, proponemos la posibilidad de utilizar la lógica de una circularidad dinámica. Esta se basa en una lógica que es posible sintetizar de manera secuencial de la siguiente manera: causa, efecto, causa. El diferenciar estos tres momentos, o comunicaciones entre el estado y la administración, requiere también considerar la administración y el Estado como ámbitos, esferas o sistemas independientes uno del otro, y por lo tanto con capacidad de tomar decisiones independientes y que se influyen mutuamente. En este fenómeno aparece una relación mucho más compleja de tipo circular, en la que intervienen de manera dinámica el Estado y la administración, y de nuevo el Estado, o en la perspectiva administrativa, el orden secuencial sería, la administración, el Estado y la administración, en esta sucesión: práctica administrativa, decisión política, nueva normatización. En este planteamiento es posible extraer un aprendizaje. No es posible formular aquí una única causa y un único efecto, sino que es posible referirse a múltiples causas y múltiples efectos, y que, a su vez, estos efectos han provocado otras causas en la diseminación de la pandemia.

La idea de circularidad - "circulación dinámica»-, ya fue delineada en la presentación constructivistasistémica de Niklas Luhmann (1993). En el ámbito de la ciencia política, consiste básicamente en el tránsito de la jerarquía de diferenciación bidimensional (arriba-abajo, o autoridad/súbdito; gobernante/gobernado), o de la presentación en la perspectiva top-down a la bottom up, a una circularidad en la que el poder político ha perdido su carácter simétrico jerárquico de arriba abajo, hacia otra perspectiva caracterizada por una diferenciación tridimensional (Política, Administración, Público). Cada uno de estos ámbitos es autónomo, y por lo tanto internas, con capacidad de crear sus propias estructuras (autorreferenciales) en sus operaciones internas. No se trata de sistemas estáticos o cerrados, sino que se encentran en constante proceso de transformación y comunicación con su entorno y con otros ámbitos. Este tipo de descripción coincide con el 
esbozo de sociedad policéntrica, o de la existencia de centros múltiples no jerárquicos entre sí. La principal característica de esta presentación es la pérdida de poder del sistema político, de modo que este se orienta cada vez más hacia los entornos creados en su interior ${ }^{2}$.

Fenómenos políticos recientes han sido analizados a menudo en la lógica causa-efecto (transición democrática española, colapso de los países del «socialismo real», primavera árabe, emergencia de los regímenes alocráticos, etc.). Han sido pocas las publicaciones que han identificado que se trataba de procesos complejos, distantes de ser lineales e irreversibles. En este estado de cosas, una serie de causas producen unos efectos a un ámbito o sistema de la sociedad, y desde este surgen nuevas causas. En la pandemia de Covid-19 se han resaltado el origen zoótico, en el que su surgimiento hipotético sería en el llamado «mercado húmedo» de la ciudad de Wuhan, y el traspaso del virus de un animal al sistema sanguíneo humano. Esta sería, según la OMS, la causa desencadenante de la pandemia.

En el plano técnico-metodológico estricto, nos basamos en un análisis de un contexto de entendimiento comunicativo, en el que las comunicaciones son simbólicas, y que incluyen el conjunto de normas, disposiciones y leyes, acciones y comunicados políticos o institucionales.

\subsection{La sociedad compleja}

La complejidad expresa un estado de cosas autocondicionado, es decir, una situación en la que sus elementos se constituyen para converger en una unidad superior a la función de un ámbito o de un sistema. La idea de complejidad que presentamos no se refiere a un conjunto de problemas complicados cuya solución requiere de múltiples variables, o a la existencia de diversos elementos interrelacionados. Es posible describir la complejidad como un exceso de relaciones, una apertura a infinitas relaciones posibles que para poder diferenciarse entre ellas requieren una operación selectiva y conectiva por parte del observador. En otras palabras, significa que en los actuales fenómenos, problemas, situaciones que aparecen constantemente, es necesario realizar una selección (conectiva) de la multiplicidad de variables en las que intervienen.

El asumir el concepto de complejidad, permite por un lado, trabajar en un plano de abstracción para poder identificar con claridad y entender lo que no se puede entender.

en un momento dado al converger simultáneamente innúmeras variables. Por otro, establecer la relación entre el ámbito o el sistema y su entorno, entendido este como la totalidad de la sociedad, otros sistemas parciales. Con estas operaciones el observador selecciona y limita determinados elementos o variables, de tal forma que algunos formen parte de un sistema y se margine necesariamente a otros.

\subsection{Complejidad, contingencia y riesgo}

La complejidad en las sociedades modernas actuales formadas por una extensa y densa organización político-administrativa, jurídica y económica en múltiples niveles, significa abordarlas necesariamente en la perspectiva de la contingencia y el riesgo. Estas sociedades se orientan cada vez más a un tipo de articulaciones, convenciones y mediaciones hacia sistemas e interacciones altamente complejas. La articulación entre los diferentes niveles de gobierno y de las instituciones, el funcionamiento de las autarquías, la gestión de las demandas y emergencia de conflictos, en las regiones y las áreas metropolitanas, las relaciones internacionales, son cada mes vez más caracterizados por largos procesos hipercomplejos en muchas ocasiones con actores con intereses diametralmente opuestos. Ello da lugar al surgimiento latente del conflicto social. El presentar la sociedad como hipercompleja presenta desafíos, ya que complejidad comporta contingencia y contingencia, inevitablemente riesgo, y riesgo a su vez, peligro: ¿la decisión sobre un tipo de desconfinamiento puede asegurar una disminución de la pandemia?, decretar un confinamiento obligatorio, ¿puede ser efectivo si son obligados a acudir a su puesto los «trabajadores esenciales» con el transporte público?.

La pandemia del Covid-19 ha puesto de manifiesto la hipercomplejidad en todas sus dimensiones: paradojas entre la economía, la salud y la ciencia (¿Por qué se paralizan unos sectores mientras que otros son obligados a acudir al trabajo como «servicios esenciales»?) paradojas y contradicciones en las decisiones en diferentes niveles de gobierno (gobernanza multinivel en países de arquitectura federal o descentrali-

2 En palabras de Luhmann: «el público influye a la política a través de las elecciones. La política establece límites y prioridades a las decisiones de la Administración (...). La Administración se vincula a sí misma y al público por sus decisiones, y este último a su vez reacciona frente a las decisiones a través de las elecciones políticas y mediante otro tipo de expresiones. N. Luhmann, 1993, pp. 63-64. 
zada), diversas lógicas de combate a la pandemia (irracionalidades en la conducta individual y colectiva preventiva y en las decisiones gubernamentales) articulación multinivel (entre los niveles de gobierno y la Organización Mundial de la Salud, etc.).

En la perspectiva que adoptamos en este escrito, se utiliza el término de complejidad como un concepto pluridimensional, al que se la atribuyen básicamente dos significados. En el primero, la complejidad es el punto de vista que expresa con mayor fuerza las problemáticas de la investigación en la moderna teoría de los sistemas, al no poder ser definida sin la existencia de los sistemas. Así, la noción de complejidad ha asumido una función que puede ser definida de catalizadora. En este sentido, la complejidad viene dada por las operaciones de selectividad de los propios límites del sistema. Estos límites deben considerarse también como dispositivos especiales para la función de acoplar y separar acciones en forma de ámbitos de acción y responsabilidades, y mediante ejecuciones específicas de selección. La selectividad propia de la ubicación de límites y de las demarcaciones de frontera de cada sistema, no sólo sirven para reducir la complejidad interna del sistema, sino también la complejidad externa. Para evitar indeterminación de los sistemas es necesaria la existencia de una racionalidad selectiva entre estos sistemas. La operación provoca que los sistemas parciales (economía, jurídico, político, administrativo, entre otros) sean indeterminados entre sí, siendo la comunicación necesaria para su regulación.

En el segundo, la complejidad organizada solo tiene lugar en la formación de sistemas, y en sus comunicaciones y códigos utilizados, al significar complejidad también en las relaciones selectivas entre los sistemas. En la óptica autorreferencial, al aumentar el número de elementos dentro de un sistema, o que un sistema forme parte del entorno de otro, puede llegar al extremo de no poder relacionar todos los elementos. Aquí aparece el concepto de complejidad, en su presentación de complejidad organizada. Así pues, la complejidad es como un estado de cosas autocondicionado, puesto que sus elementos deben constituirse completamente para converger como unidad en el nivel superior de su formación, ya que en los sistemas su capacidad de acoplamiento es limitada.

\subsection{Hipercomplejidad y Administración pública}

Todavía es reciente la idea de que los procesos de toma de decisiones, la gestión en las políticas públicas, los procesos administrativos y burocráticos de la administración pública son procesos complejos ${ }^{3}$, siendo considerados como un «becoming field» (Eppel y Lee, 2020). A diferencia de otras ciencias sociales, como es la economía, es todavía reciente la utilización de las ideas de la complejidad en las observaciones de la administración y gestión pública. Los enfoques vinculados con la complejidad y los enfoques evolutivos y sistémicos en la perspectiva autorreferencial, han recibido poca atención analítica y empírica. La pregunta que surge es: ¿Cómo puede contribuir esta teoría, o punto de partida de la complejidad para aumentar la compresión y las propuestas de resolución de problemas en la administración? Algunas publicaciones recientes examinan las aplicaciones de complejidad (Pont-Vidal, 2021; 2017; Mitleton-Kelly; Teisman et al., 2009) y focalizan las posibilidades de esta teoría en un ámbito general, los procesos de toma de decisiones y las redes de gobierno o los sistemas de gobernanza. En ámbitos económicos específicos (hospitalario, empresarial), las publicaciones han hecho hincapié en el potencial de la teoría de la complejidad para abordar el desarrollo de políticas (Tait y Richardson, 2009; Morçöl, 2012). Otros trabajos incluso cuestionan el papel de los expertos de políticas y su pretendida «expertise» «autoridad» y «experiencia» de los modelos económicos tradicionales, al tratarse de un simple reduccionismo, puesto que ignoran el complex thinking y la existencia de una lógica de pensamiento no lineal.

La teoría de sistemas autorreferenciales permite la descripción holística de estos fenómenos para facilitar la posterior toma de decisiones. Sin embargo, es necesario abordar algunos matices, puesto que son diversos los autores para utilizan esta perspectiva aunque con diferente significados. Teismann et. al (2009) ha respondido a esta pregunta a partir del supuesto de los principios de la good governance y de la capacidad dinámica de autoorganizarse, como una característica permanente en evolución en los procesos emergentes. Aunque no asume la lógica de la capacidad operativa autónoma de un sistema, y por lo tanto, de crear sus propias estructuras, estos procesos se forman a partir de eventos, fenómenos de acción e interacción colectiva y los cuales contribuyen a formar una estructura institucional. Así, la autoorganización surge de la capacidad de

3 Como Administración pública nos referimos, en el caso de España, a la Administración General del Estado, las Administraciones autonómicas y la Administración local. 
libre elección y acción de las personas participantes y las organizaciones, en el sistema político o económico. No obstante, la interacción también puede obstruir estos procesos, por lo que es necesaria un tipo de comprensión de la coordinación, ya que la integración difiere de las ideas convencionales sobre la adaptación mutua. La sincronización entre los participantes o las organizaciones, presupone que una estructura es insuficiente para estimular la integración, aunque existe un contexto de diversidad institucional (política, jurídica) dentro del cual los límites cambian constantemente, que requiere que se establezcan nuevas interconexiones entre ellos, como ha sucedido en los problemas de gestión entre el Gobierno y las Comunidades Autónomas.

A partir de ejemplos y de casos prácticos, estos trabajos han focalizado los problemas que surgen en la gestión en el contexto de las exigencias que la complejidad impone, sin entrar en los cambios en la administración, y en su relación con el Estado y el sistema político. Por otro lado, en sus referencias sistémicas aparecen paradojas conceptuales no resueltas Estas teorías convergen en la noción de complejidad, aunque con diferentes supuestos. La contingencia está presente en la administración -como se ha mostrado con la pandemia - puesto que muchas acciones no eran necesarias, aunque a pesar de ello los efectos de la pandemia han estado presentes. La administración ha tenido que adaptarse a este entorno con respuestas también contingentes: ¿Cuál ha sido el confinamiento más eficaz para combatirla, el inteligente, o el obligatorio? Esta presentación se refiere a la descripción binaria de sistemas abiertos, en cuya lógica input-output, el sistema se relaciona de manera pasiva y dependiente con su entorno.

\subsection{Contingencia, riesgo y observación}

A pesar de la advertencia de la Organización Mundial de la Salud en el año 2019 sobre de la posibilidad de una pandemia mundial causada por un virus desconocido, ningún gobierno se preparó para esta situación. Con la eclosión del Covid-19 en Wuhan, el mes de enero del 2020, la OMS minimizó la posibilidad de una pandemia de alcance mundial. En un primer momento, esta organización admitió el 27 de enero, un error en la divulgación del riesgo global del nuevo coronavirus, y calificó el riesgo de «moderado», siendo rectificado más tarde por «alto», y posteriormente se informó que el riesgo es «muy alto en China, alto regionalmente y alto globalmente». La imperceptibilidad de los peligros, su supranacionalidad, la "expropiación ecológica», y el paso de la normalidad a la absurdidad ya fueron diagnosticados por Beck ([1986] 1998), con ocasión del accidente nuclear de Chernobil en 1986. La contingencia estuvo presente en las decisiones de la OMS: ¿Por qué no fue necesario informar del elevado riesgo?, ¿Era imposible un riesgo «moderado»? ¿Podía ser que fuera tan rápida su expansión e infección? ¿Por qué permitió e régimen chino los vuelos internacionales sabiendo de la rapidez del contagio?

Como la contingencia, el riesgo y el peligro constituyen una secuencia dinámica y continua ha quedado claro en la gestión sanitaria. Contingencia, en la perspectiva que presentamos aquí, es aquello que no es necesario ni imposible, pro que pude existir siempre, comporta necesariamente riesgo, y el riesgo compota a su vez peligro. Para Luhmann, además de los predicados ontológicos «ser» y «no ser», la contingencia también implica un tercer valor, a saber, la «indeterminabilidad» o incerteza. La era de incertidumbre contemporánea no es más que una versión particular de la sociedad moderna, cuyo atributo definitorio es la contingencia. En las condiciones modernas, las necesidades y las imposibilidades ya no proporcionan el marco ordenado del mundo. Ya no existen formas socialmente necesarias para la limitación recíproca de los diversos componentes de la sociedad o incluso para la coherencia social del conjunto. Se acepta que todo lo que se supone puede ser diferente, y que cualquier cosa, aunque no todo de una vez, se puede redefinir y cambiar a través de la comunicación. La sociedad moderna es una sociedad que abarca las contingencias como ninguna otra antes. Si bien las necesidades e imposibilidades se aceptan por razones de tiempo, este tipo de sociedad, se caracteriza sobre todo por atributos débiles requeridos en el sentido de propiedades emergentes inicialmente indeterminables, altamente improbables y frágiles. El inicio de los movimientos políticos populistas minoritarios, han logrado vencer elecciones democráticas y proclamar a presidentes (sólo en Europa, Viktor Orbán en Hungría, Vladimir Putin en Rusia, y modificar constituciones democráticas y el poder judiciario, en Polonia). Debería ser obvio que, en las condiciones contemporáneas, tanto la ciencia como la política, así como su relación entre sí, ya no pueden concebirse en términos de necesidad, sino que deben analizarse en términos de estas propiedades improbables y frágiles emergentes de sociedad pueden ser posibles en cualquier momento. Al mismo tiempo, sin embargo, debe recordarse que, en estas condiciones, nada se considera imposible (accidentes en plantas nucleares o de máxima seguridad).

Las diferentes corrientes en la filosofía y en las ciencias sociales han atribuido al término de riesgo diversos significados. En la tradición racionalista ha sido considerando como producto de una decisión que 
según sus impactos posteriores (negativos) se hubieran podido prever o evitar. Aquí se trata de un cálculo temporal con el horizonte de un futuro incierto, y cuyos impactos podrían ser evitados. Pueden ser aceptadas situaciones de riesgo que lo contemplen, siempre y cuando esté justificada la posibilidad de que ocurra (construcción de grandes represas, empresas contaminantes, experimentación con nuevos virus, etc.). Esta ha sido la argumentación imperante. Se ha cuestionado el concepto tradicional de contingencia por ser limitada, puesto que solo puede describir riesgo en sus aspectos cuantitativos y probabilísticos, y los analiza básicamente en el sistema económico y científico. Tampoco la observación de primer grado basada en códigos binarios formulados bajo presentación estadística, permite el análisis en profundidad de las lógicas de razonamiento del ser humano, que tampoco calcula como debería ser el predicado y la acción racional, por lo que comete constantemente errores.

El abordaje y observación del riesgo en la diferenciación entra la observación elemental y la observación de segundo grado. En este tipo de observación, el mundo real y material es mesurable, y por lo tanto, limitada a poder solamente identificar cuantitativamente los posibles riesgos. Con la utilización del concepto binario riesgo-seguridad, se trata de observaciones solo para garantizar la seguridad, para la cual es necesario más y mejor tipo de información. Por esto se propone que la teoría debe ser orientada a la observación de segundo orden. Se trata de un fenómeno de "contingencia múltiple», que permite y ofrece abordarlo desde diferentes perspectivas por diferentes observadores. Aquí el problema reside en que el riesgo algo que es descrito como similar por diferentes observadores cuya consecuencia es que genera informaciones muy diversas entre ellos. En este punto, aparece la diferenciación entre riesgo y peligro. Mientras que el riesgo se refiere al caso en que el desafío es consecuencia de la decisión previa, con el peligro los posibles daños son provocados externamente, es decir atribuido al entorno del sistema. La observación de segundo grado, permite establecer la distinción entre quienes deciden que son los riesgos y quienes son los afectados. ¿Quién definió el tipo de confinamiento, para que tipo de población?

La economía es el ámbito en el que habitualmente se ha considerado el riesgo (mitigándolo mediante seguros). Aquí los riesgos son aquellos que tienen que ver con diferencias temporales en la utilización del dinero (vinculados con la inversión y el crédito): una falla en la producción es considerado por el como un peligro, no como un riesgo. Así para la economía el riesgo central es el de la no reconstitución de la capacidad de pago. La observación de segundo orden está orientada a la observación del cálculo en el sistema económico, y focaliza que la competencia y el afán por mantenerse en el mercado lleva a los agentes económicos a observar no la economía, sino cómo se comportan los competidores para reproducir su propia conducta. En un sentido subjetivo, el riesgo se trataría de una especie de coacción que impulsa a la imprudencia, mientras que, en el sentido social, se trata de un aumento en la disposición al riesgo (coeficiente de endeudamiento, el endeudamiento global). En suma, en la observación de segundo grado las cuestiones están orientadas a: ¿Qué es, o debería ser, una administración orientada para la ciudadanía? ¿Cuál es la lógica de la toma de decisiones administrativas? ¿Qué tipo de administración queremos?

\section{ADMINISTRACIÓN PÚBLICA}

Los impactos de la pandemia indican que el periodo «entre dos épocas» ha terminado de repente, y de forma que nadie lo esperaba de esta manera, dando comienzo una nueva época, caracterizada por ser en muchos aspectos contingente y vinculada a la incerteza. No es impulsada por un sujeto revolucionario, sino por un micro organismo: el Covid-19. En alcance, su rapidez en la extensión, y sus trágicos impactos en el ámbito humano, ha tomado a todos los países y sociedades por sorpresa. En esta situación inesperada, los Gobiernos han actuado por medio de medidas drásticas que han abarcado desde el confinamiento general de la población, hasta el confinamiento inteligente o selectivo.

No nos detendremos aquí a analizar los problemas heredados de las administraciones, ya abordadas por diversos estudios ${ }^{4}$. Durante la pandemia, ya fueron puestos a prueba mecanismos y técnicas de comunicación información (TICs) prestación de servicios de la administración, algunos ya existentes y utilizados (administración Gob.es), otros infrautilizados (videoconferencias) y otros más novedosos. La pandemia ha obligado a agilizar en carácter de urgencia las formas de trabajo de las administraciones y adaptarlas a unas

4 Jiménez Asensio, 2020 identifica sus problemas estructurales («visión cortoplacista de la política», «marco normativo obsoleto», envejecimiento de las plantillas, etc.). 
circunstancias excepcionales: su organización se ha tenido que extender medidas incipientes antes (flexibilización, reorganización de turnos, teletrabajo, homework).

Ante la grave crisis económica, laboral y social que está viviendo el país y en el contexto de la reformulación de las responsabilidades y extensión del Estado, se perfilan y presentan diferentes desafíos para la administración en el futuro. Es posible perfilar dos modelos principales:

Primero, en un contexto de transformación de las responsabilidades del Estado -ya iniciado unos años antes- y de su relación con la sociedad. Según el modelo de actuación del Estado que finalmente se afiance (que varía entre un Estado más amplio a otro más reducido) ambos vinculados con procesos de recentralización, la administración se verá afectada en su autonomía, capacidad y ámbito de acción, además de sus formas tradicionales de planificar el trabajo. La actuación de los gobiernos durante la pandemia ya ha apuntado claramente en estas direcciones. Esta situación ya fue diagnosticada conceptualmente unas décadas atrás, y reformulada en los últimos años.

Segundo, con el modelo de Estado se inician posibilidades de introducir, o de acelerar cambios estructurales, y la generalización de las posibilidades de las tecnologías de información y comunicación ya iniciadas (e-government, e-administration, e-democracy) y en las que se resaltan los aspectos positivos. Ello puede conducir a nuevas formas de "gobernanza inteligente», "gobierno electrónico», pero también pueden representar la implementación de nuevas posibilidades de vigilancia y control (Attour y Chaupain-Guillot, 2020). Las publicaciones sobre el tema son abundantes, en que las se exponen las posibilidades de Big data y en la extensión de la denominada "tecnolopolítica» ${ }^{5}$, aunque también se muestran los impactos negativos y escépticos en su aplicación y en la formación de una sociedad de vigilancia.

\subsection{Génesis de los análisis actuales}

Cada uno de estas dos tendencias presentadas aquí de forma esquemática corresponde a diferentes supuestos conceptuales, políticos y de gobernanza sobre la manera de entender la regulación de la sociedad en su totalidad. Ambos modelos tienen sus raíces en los supuestos del llamado movimiento de la Nueva Gestión Pública, iniciado a finales de la década de 1990, el cual ha sido ampliamente analizado y discutido entre sus defensores y sus críticos. Sus contradicciones referentes al mantenimiento de las estructuras tradicionales de toma de decisiones han servido para que las administraciones y los políticos hayan maximizado su utilidad y tendido a extender sus lógicas instrumentales y su influencia.

A partir de la década de los años 80 , aparecieron numerosas monografías que asociaron los cambios que se produjeron en la administración, en las que se vinculó su transformación con la modernización de los aspectos internos y externos, la transformación del Estado en sus funciones y extensión, bajo el movimiento conceptual político y económico del New Public Management y las nuevas corrientes de la gerencia púbica (Anter, 2012; Hilgers et al. 2012). Este movimiento se inició en consonancia con la ya diagnosticada crisis del Estado de bienestar, y los preceptos económicos del Consenso de Washington. Los postulados de la Nueva Gestión Pública corresponden a un delineamiento de la relación administración-Estado, siendo el impulso para la renovación de las administraciones públicas durante el último cuarto de siglo. No obstante, la NGP no ha estado libre de controversias, destacando entre estas las corrientes neo-marxistas y la perspectiva conservadora de la gestión. Entre los primeros destacan las críticas en la perspectiva del modelo de Estado, dirigiéndose a su vínculo demasiado estrecho con la economía, al promover el desmantelamiento y reducción del Estado, ser «hostil al Estado» y desmantelar los cimientos de la democracia participativa o representativa liberal. Para los conservadores, la NGP ha intervenido demasiado en la regulación de la administración. En el problema irresoluble que se constata en el vínculo Estado-economía, o administracióneconomía surge de la paradoja, que mientras el Estado debe estar orientado al bien común, la economía buscará siempre la maximización de los beneficios. El análisis del vínculo recíproco Estado-administración, como dos ámbitos o esferas separadas, aunque vinculados a partir de políticas regulativas, y fue descrito y abordado en base a la transformación de la administración (Döhler, 2006; Hilhers et al., 2012).

La Nueva Gobernanza Pública ha reemplazado gradualmente a las organizaciones de servicios dispersos (asociaciones, fundaciones, Tercer sector, cooperativas de servicios) por un entramado de redes de

5 Bajo el término «tecnopolítico» comprende la política democrática, es posible distinguir dos corrientes principales: estudios sobre políticas mejoradas en Internet, gobierno electrónico y Política 2.0 para facilitar las prácticas existentes (votación electrónica, campaña electrónica y petición electrónica). Por otro lado, la perspectiva habilitada para Internet se basa en la idea de que las TIC son esenciales para la organización (u organización de) políticas como la participación ciudadana y procesos deliberativos. 
sistemas de prestación de servicios públicos, cuyo funcionamiento se basa en la interacción de una multiplicidad de actores para lograr los objetivos sociales y la prestación de servicios públicos con énfasis en la asociación y la colaboración. Se trata de una adaptación del modelo institucional jerárquico (top down) tradicional, hacia un modelo de inspiración compleja para permitir abordar las necesidades sociales y también como instrumento para la inclusión social. La coproducción de servicios ha adquirido una nueva dimensión y adquiere una importancia central de la producción de servicios públicos. En pocos años, la administración ha experimentado una evolución y transformación con impactos en su conceptualización tradicional, al pasar del concepto de usuarios de servicios en la planificación y prestación de servicios públicos, al concepto de clientes y consumidores (no libre de críticas), y por último al concepto de ciudadanos y coproductores de servicios.

Las causas de esta transformación son diversas. El modelo de Estado y su gobernanza, han sido objeto de exhaustivos análisis básicamente en dos perspectivas. Por un lado, su crisis estructural fue abordado por la perspectiva neo-marxista y sus variantes ${ }^{6}$, y en la relación entre las instituciones estatales y el sistema político, mientras que el análisis del desarrollo, modelos y posterior crisis del Estado de bienestar, fueron el foco de numerosos investigaciones e interpretaciones en la mayoría de democracias europeas (Jürgen Habermas, Niklas Luhmann, Esping Andersen; Josep Picó, entre otros). A mediados de la década de los años 90 , se inició el análisis con la presentación de la complejidad y del supuesto de la presentación de la sociedad policéntrica (Luhmann, 1993; Schimank, 2009; Willke, 1996; 1993).

La Gran Recesión que experimentaron los países occidentales en el año 2008, condujo a que los análisis y publicaciones se orientaran a los aspectos relativos a la capacidad (o incapacidad) regulativa y orientativa del Estado (Veit et al., 2019) y al fracaso de la gobernanza y de las ciencias contables para predecir la crisis que se avecinaba bajo el laissez faire de la economía. Frente a la gravedad y profundidad de la crisis desencadenada se cuestionó la capacidad idealizada y autorreguladora del mercado, y la necesidad de incluir la democracia como elemento fundamental en la presentación de la composición de la sociedad (Pont Vidal, 2019a, 2019b, 2018a, 2018b, 2018c), y las posibilidades de cooperación y coproducción de servicios a partir de una nueva visión administrativa (Döhler y Wegrich, 2010; Sevilla et al. 2010).

\section{2. ¿Dónde nos encontramos hoy?}

En la segunda década del siglo actual, se produce una clara ruptura con los supuestos, surgiendo dos tendencias asimétricas. Por un lado, las corrientes que pretenden seguir profundizando en la coproducción participativa de servicios públicos. Por otro, con un profundo cuestionamiento de las limitaciones del Estado de bienestar, ya iniciados tres décadas antes, los cuales han producido una reacción para el retorno a la privatización de «lo público» en la que se hace responsable a cada ciudadano sobre su situación y por lo tanto sobre su capacidad de solicitar servicios personales en las administraciones. Esta orientación adquiere su forma política en diversos países, con la emergencia de partidos de extrema derecha o conservadores al poder (Gran Bretaña, Norteamérica, Brasil, Hungría, Polonia). Solo en unas publicaciones aisladas se estableció el nexo entre la sociedad y el Estado y la interacción entre estos desde la óptica de sistemas autorreferenciales (Luhmann, 1971; 1983) y en sus variantes ontológicas (Mayntz, 1997; Scharpf y Mayntz, 1995), o el vínculo con los presupuestos públicos (Pressman y Wildavsky, 1973), ha abordado como ámbitos separados.

En la perspectiva de sistemas autorreferenciales, la administración la describimos como un sistema dinámico y autónomo, con una diferenciación dinámica de la burocracia, formado por jerarquías en estructuras asimétricas, que se manifiestan en las jerarquías y en la utilización del poder por parte de fuerzas políticas. La función sociopolítica de la administración exige una racionalidad que significa un grado de diferenciación dinámica, o sea, capacidad de autodeterminación de su comportamiento. Como sistema con una organización funcional específica, tiene la función de producir decisiones vinculantes sobre problemas bajo los supuestos de una complejidad ya previamente reducida por el sistema político. En este sistema ya han tenido lugar los procesos políticos en lo que se elabora la información, cuyos resultados se transforman en inputs para la administración como sistema.

Las decisiones se constituyen con la ayuda de la política, con lo que el sistema puede alcanzar un elevado grado de complejidad y con capacidad de decisión. Luhmann ([2010] 2014) delinea dos tipos de complejidad: la complejidad indeterminada y la complejidad determinada. Mientras que en la primera la po-

6 Offe, 1974. 
lítica tiene un papel determinado en la administración al direccionarla según sus prioridades políticas, en la segunda este presupuesto no es satisfecho al acentuarse su autonomía respecto a la política, siendo que la administración depende de un entramado de vínculos con la sociedad. La autonomía de la administración puede distinguirse tres dimensiones. La social, referente a los objetos con los que actual, la objetiva, en el sentido de sus decisiones y la temporal, en los procesos de decisión.

La mayoría de las predicciones y prospecciones realizadas en los últimos años sobre la administración, respecto a la extensión y responsabilidades del Estado han quedado en su mayoría ultrapasadas, mientras que otras se verán reforzadas por la rapidez e impactos de los acontecimientos derivados del coronavirus, y otras han ganado en actualidad, así como delineamientos de una gobernanza sistémica en la que los subsistemas (economía, política, jurídico) interactúan de manera más eficaz, con una predominancia del sistema científico.

\subsection{Escenario post Covid-19: modelos auto-excluyentes}

Se coincide en que el escenario pos-virus abre incertezas y contingencias. Asumir la contingencia no significa instalarse en un pesimismo, o incerteza continua, sino que abre también nuevas posibilidades. Es evidente que habrá que explorar los posibles cambios del Estado y de la administración desde la perspectiva de la alta complejidad, o hipercomplejidad, que caracteriza las sociedades modernas. Complejidad en el sentido sistémico, significa contingencia, y contingencia significa, riesgo y peligro. Viene a ser pues, que algo no es ni imposible ni necesario pero que pude ser. Esta doble negación se presta a ambigüedades y por lo tanto a interpretaciones diversas. Para la idea que presentamos en este texto, posee el valor de que cualquier propuesta de cambio será posible, y por lo tanto, podría ser efectiva. La cuestión importante para nosotros es: ¿en qué dirección se producirá este cambio? Aquí aparece el gran desafío para las democracias liberales el Estado y la administración.

Ha sido repetido que la intención por parte de los gobiernos de confinamiento de la población (horizontal, vertical, «inmunidad del rebaño», selectivo o inteligente), ha obedecido a la lógica excepcional de evitar el colapso del sistema de salud de cada país. Para evitar el colapso, o la posibilidad de no poder atender a todos los casos necesarios en una fecha, es necesario que el número de contaminados no sobrepase en ningún momento un máximo que el sistema sanitario pueda gestionar y atender, ha sido necesario que la curva de contagios necesitados de atención médica se mantuviera lo más plana posible, durante el espacio de tiempo más extenso, en lugar de una curva de rápida ascensión y durante poco tiempo, hasta alcanzarse el llamado "pico» de los casos atendidos. Por otro lado, esta estrategia también ha proporcionado al sistema científico más tiempo para el desarrollo de un tratamiento, o del desarrollo de una vacuna. El confinamiento vertical ha correspondido a la lógica de evitar al máximo la paralización económica de los países afectados, con el argumento, si para la economía los impactos de la pandemia serán peores, a destruirse la base de la riqueza y el empleo en el país. La población destinada al confinamiento era la población de riesgo (co más de 60 años, o con enfermedades crónicas). En la fase inicial de la pandemia fue recomendado por los gobiernos de Gran Bretaña, Norteamérica, y Brasil y Suecia, aunque a partir de una perspectiva inicia diferente. Por otro lado, el confinamiento horizontal, ha implicado a la mayor parte de la población. Fue decretado en Italia y España. En Alemania, se ha mostrado como el más efectivo, al ser el país europeo con menos fallecimientos, aunque por diversas razones. ¿A qué lógicas político-administrativas y de entendimiento del estado corresponde cada modelo?

En este estado de cosas. dos modelos se perfilan: 1) Estado reducido y continuidad económica; 2) Estado extenso, vinculado con la presentación de un Estado-céntrico. Los dos modelos ya es posible identificarlos en diversos países, y se seguirán ampliando en el futuro próximo. En la perspectiva de la hipercomplejidad, aparecen paradojas en los dos modelos. La autonomía del sistema administrativo respecto a la política puede suponer dos fenómenos diferentes. Por un lado, no estar sometido al control interesado del sistema político, y por lo tanto favorecer su función elemental de administrar. Por otro, puede adquirir una preponderancia respecto al sistema político por lo que puede quedar sin ningún tipo de control político. Hemos realizado un esbozo de delineamiento que corresponde a dos lógicas: «Estado reducido» y "Estado extensivo (o Estado-céntrico»). Ambos modelos no están libres de paradojas.

\subsection{Estado reducido jerárquico}

La propuesta de reducir el tamaño y reformular las funciones del Estado no es nueva, manifestándose en el plano político recientemente en un contexto de crisis de la democracia liberal, la emergencia de 
partidos y movimientos populistas radicales, y la substitución por regímenes alocráticos. Puede adquirir dos formas diferentes: una organización del Estado centralizador de carácter centrípeto y una organización policéntrica. En el contexto de crisis económica (impactos de la Gran Recesión del año 2008), se pretende reducir niveles decisorios en el plano multinivel, eliminando estructuras que puedan molestar la toma de decisiones rápidas. Desde hace unos años, ya viene implementándose en Norteamérica, con la priorización de la responsabilidad individual en todos los ámbitos (educación, sanidad, empleo, jubilación), lo que significa que en situaciones de riesgo y peligro social el estado no será responsable. El sistema económico debe ser dejado a la iniciativa privada con la mínima intervención del Estado. La gestión de la pandemia de coronavirus ha mostrado la contingencia de este modelo. Se podía haber declarado el confinamiento vertical, si hubiera existido un sistema sanitario con capacidad para atender a toda la población con calidad para una situación excepcional como es esta pandemia. El asumir un confinamiento vertical, viene a significar que el país debería disponer de un sistema de salud exponencialmente más extenso y amplio para la población (en principio para sin exclusión para situaciones de emergencia como la actual) en los niveles cuantitativo y cualitativo. Con la situación creada por esta pandemia también han emergido sus paradojas en el ámbito político al converger movimientos ciudadanos radicales (entre los que se encuentra la extrema derecha) las libertades civiles, frente a unos métodos impuestos de control, vigilancia y confinamiento decretados por los gobiernos.

\subsection{Estado extensivo: Estado-céntrico}

La pandemia ha servido también para que diversas publicaciones puedan predecir el esperado colapso del sistema de libre mercado o capitalista. Sus propuestas abarcan desde visiones apocalípticas del futuro (exclusivamente imaginativas) con hasta predicciones realistas. Para sectores políticos de izquierda, el confinamiento ha poseído una virtud, y predicción: con la ampliación temporal de la crisis del coronavirus, también cambiará en gran medida el orden económico. El horizonte y la perspectiva es que la economía de mercado tal como se conoce probablemente se abolirá parcialmente, en la que la propiedad privada y la competencia serán menos importantes, y la influencia del Estado aumentará masivamente.

Se retoma así la teoría Estado-céntrica, con un Estado como principal productor de bienestar social, que también puede significar paradójicamente mayor control, burocrático y centralización administrativa. La paradoja emerge aquí con el tipo de Estado deseado: ¿Un Estado opaco y burocrático de control eficiente en las situaciones de emergencia pero fiscalizador de la ciudadanía y de los medios de comunicación?

En ambos modelos aparecen pues paradojas, puesto que un Estado reducido puede favorecer formas de gobierno como autogobierno, autoorganización, autorregulación, o glocalización, mientras que un Estado extenso tendería a la absorción de iniciativas de este tipo, en pro de acciones estatales centralizadas y homogenizadoras, en un contexto económico globalizador. Esta disyuntiva aparecería en estados de orden constitucional federal y también en los de organización descentralizada.

\section{CONCLUSIONES Y UNAS REFLEXIONES}

En la línea argumentativa conceptual y metodológica expuesta en este trabajo, la presentación de la sociedad compleja (o hipercompleja), ofrece un enorme potencial heurístico-constructivista para mejorar la identificación y la comprensión del Estado y la administración en el contexto contingente actual. Presentar la complejidad de las relaciones, conexiones y decisiones en las sociedades modernas actuales requiere también asumir los conceptos de autoorganización, no linealidad y circularidad de los fenómenos, y las nociones de riesgo, coevolución, capacidad conectiva, emergencia, estructuras intermedias. El contexto descriptivo se enmarca en la contingencia y la incertidumbre, en la que existen posibilidades de cambio en las relaciones entre Estado y sociedad, aunque estas no serán necesarias como tampoco imposibilitadas de un nuevo tipo de gobernanza, si bien siempre bajo la posibilidad y la incertidumbre. Este tipo de sociedades y de transformaciones del Estado, ya emergidas desde unos años, la pandemia de Covid-19 las ha acelerado, de tal forma que es posible referirse a un nuevo tipo cualitativo emergente de sociedad.

La óptica de sistemas autorreferenciales, se construye como una compleja estructura comunicativa que sobrepasa los paradigmas procedimentales centrados en la acción comunicativa para lograr el consenso, y con los paradigmas esencialistas que se han utilizado habitualmente para la observación en las teorías del Estado. Los paradigmas de interacción habituales no han permitido identificar una clara diferenciación sobre 
la manera en que los mecanismos de decisión son aplicados, y como tiene lugar una paulatina pérdida de autonomía entre los subsistemas o sistemas parciales de la política, la administración y la ciudadanía.

En el ámbito de la reorganización de la extensión y modelo de Estado y su modelo de gobernanza, emergen diversas orientaciones, desde el Estado reducido jerárquico, a una orientación Estado-céntrica. A cada una de estas orientaciones corresponden diversas estrategias tampoco libres de paradojas, hasta otras que aspiran incluso al esperado fin del capitalismo por los sectores populistas y radicales. ¿Un Estado extensivo no puede ser también un Estado de control y de rápida y acrítica implementación del Big data? Por el contrario: ¿Un Estado reducido no posibilitará que los sistemas funcionales que lo componen se conviertan en núcleos de poder independientes y autocráticos en competencia entre estos, dificultando así la gobernanza de la sociedad?

La administración como un sistema autónomo, se verá afectada por los cambios procedentes de su entorno, acelerados de manera rápida por la nueva situación. Las soluciones propuestas no amagan importantes brechas de seguridad y la insuficiente protección jurídica de los ciudadanos frente al Big data y a las TICs. La Administración asume los límites y prioridades de la política. Sin embargo, una dependencia de esta puede ser contraproducente teniendo en cuenta las recientes orientaciones populistas generalizadas (de derecha y de izquierda) de la política, La administración deberá mantener su autonomía basada en criterios técnicos en todos sus niveles, en especial en los superiores de dirección. En el plano operativo, la administración pública dejará su aparente área de comodidad (jurídico, económico, político) para entrar en la incertidumbre. Implica un abanico de posibilidades que comprenden desde la pérdida de autonomía, el incremento de la burocracia, o la derivación hacia modelos opacos y poco transparentes, con escasas posibilidades de innovación ciudadana, o en su caso de innovación controlada por una burocracia extrema.

En este escenario, un aumento de la burocracia en el ámbito político vendrá a suponer un tipo de comunicaciones opacas con una deficiente transparencia y opacidad caracterizadas por sus códigos autoritarios. La burocracia se verá ampliada y extendida con el control de la información y obsesión por la «estabilidad social». El riesgo será constante debido a la colonización por parte de la política. Esta colonización ya ha sido denunciada frecuentemente, aunque con pocos resultados concretos como se ha hecho evidente durante la pandemia, con directrices emanadas de políticos y ministros en todos los países con escasa capacitación técnica y de gestión.

La relación administración, Estado y sociedad, dependerá de varios factores: el modelo de gobernanza, la fuerza política en el poder, los modelos y prácticas de audiencias públicas que se apliquen, el tipo de cooperación público-privada, y en definitiva, la capacidad de autonomía de la administración frente al colonialismo de la política, sin criterios técnicos. El sentido, o los tradicionales horizontes utópicos, no vendrá dado aquí solo condicionado a la existencia de cosas y fenómenos, sino que será construido a partir de las comunicaciones intersistémicas. Se trata de fundamentos científicos y no metafísicos sobre la noción del sujeto, como ha sido habitual en las sociedades. Pero no será suficiente, bajo el peligro de transformarse en un sentido estrictamente tecnocrático El sentido deberá también proceder, en última instancia de otros sistemas. El sistema político, que seguirá teniendo una posición de orientación en la sociedad (la democracia será su manifestación); el sistema psíquico, que en definitiva se refiere a los pensamientos de las personas (la capacidad de pensamiento y autorrealización); el sistema de interacción, en el que las personas interactúan directamente (producido en grupos de intervención y acción ciudadana).

¿Seguirá existiendo un único centro de poder, como tradicionalmente ha sido al abordar el Estado?, ¿Será posible observar un único centro del ejercicio del poder? Este tipo de preguntas nos direccionan a la prospección, y por lo tanto de respuestas arbitrarias y vinculadas con la incertidumbre, y que requerirán necesariamente de deducciones analógicas y empíricas.

Por último, en este contexto argumentativo, es la observación y sobre la necesidad de reformular conceptualmente lo que se entiende por estado y por Administración pública, en base a una nueva lógica conceptual, basada en comunicaciones (simbólicas), y vinculada con las transformaciones de la sociedad.

\section{REFERENCIAS BIBLIOGRÁFICAS}

Anter, A. (2012). Der Staat als Beobachtungsobjekt der Sozialwissenschaften. Das Trugbild vom verschwindenden Staat und die Normativität des Gegenstandes. En M. Bach (ed.), Der entmachtete Leviathan (Zeitschrift für Politik: Sonderheft 5, pp. 15-29). https://doi.org/10.5771/9783845244273-15 
Attour, A. y Chaupain-Guillot, S. (2020). Digital Innovations in Public Administrations: Technological or Policy Innovation Diffusion? Journal of Innovation Economics \& Management, 31, 195-219. https://doi.org/10.3917/jie.pr1.0061

Beck, U. (1998). La sociedad del riesgo. Hacia una nueva modernidad. Paidós Ibérica.

Döhler, M. (2006). Regulative Politik und die Transformation der klassischen Verwaltung. Politische Vierteljahresschrift: Sonderheft, 37, 208-227. https://www.ssoar.info/ssoar/handle/document/41367

Döhler, M. y Wegrich, K. (2010). Regulierung als Konzept und Instrument moderner Staatstätigkeit. Der moderne Staat, 3(1), 31-52. https://www.budrich-journals.de/index.php/dms/article/view/3893

Engel, A. (2004). Wissensmanagement in der öffentlichen Verwaltung: Grundlagen - Potenziale - Ansätze Erfahrungen. En C. Reichard, M. Scheske y T. Schuppan (eds.), Das Reformkonzept E-Government : Potenziale - Ansätze - Erfahrungen (pp. 210-227). LIT-Verlag.

Eppel, A. y Lee, M. (2020). Complexity Theory in Public Administration. Routledge Taylor \& Francis Group.

Hilgers, D., Schauer, R. y Thom, N. (eds.) (2012). Public Management im Paradigmenwechsel: Staat und Verwaltung im Spannungsfeld von New Public Management, Open Government und bürokratischer Restauration. Trauner Verlag. Jessop, B. (2016). The State: Past, Present, Future. Polity Press.

Jiménez Asensio, R. (16 de abril de 2020). Empleo público 2020-2030 (I): Desafíos múltiples en un escenario de crisis [entrada de blog]. La Mirada Institucional. Consultado el 7 de julio de 2020. https://rafaeljimenezasensio. com/2020/04/16/empleo-publico-2020-2030-i-desafios-multiples-en-un-escenario-de-crisis-1/

Kurban, C., Peña-López, I. y Haberer, M. (2016). What is technopolitics? A conceptual scheme for understanding politics in the digital age. IDP: Revista de Internet, Derecho y Política, 24, 3-20. https://doi.org/10.7238/idp. v0i24.3061

Lenk, K., Wengelowski, P. y Meyerholt, U. (2014). Wissen managen in Staat und Verwaltung. Edition Sigma. https://doi. org/10.5771/9783845267913

Luhmann, N. (2010). Introdução à Teoria dos Sistemas. Vozes.

Luhmann, N. (1971). Opportunismus und Programatik in der Offentlichen Verwaltung. En N. Luhmann, Politische Planung: Aufsätze zur Sociologie von Politik und Verwaltung (pp. 165-180). VS Verlag für Sozialwissenschaften, Wiesbaden.

Luhmann, N. (1983). Legitimation durch Vefahren. Suhrkamp Verlag.

Luhmann, N. (2014). Sociología política. Trotta Editorial.

Luhmann, N. (1993). Teoría política en el estado de Bienestar. Alianza.

Luhmann, N. (2006). Sociología del riesgo. Universidad Iberoamericana-ITESO.

Mayntz, R. (1997). Soziale Dynamik und politischen Steuerung: Theoretische und methodologische Überlegungen. Campus Verlag.

Mitleton-kelly, E., Paraskevas, A. y Day, C. (eds.) (2018). Handbook of Research Methods in Complexity Science: Theory and Applications. Edward Elgar.

Morçöl, G. (2012). A complexity theory for public policy. Routledge.

Offe, C. (1974). Structural Problems of the Capitalist State. Class Rule and the Political System. On the Selectiveness of Political Institutions. En K. Von Beyme (ed.), German Political Studies (vol. I, pp. 31-57). SAGE.

Pont Vidal, J. (2021). Sociedades contingentes. Covid-19: un nuevo agente de cambio social. Libros de la Catatara.

Pont Vidal, J. (2019a). Teorías neosistémicas y administración pública: posibilidades observacionales y regulativas. Revista del CLAD Reforma y Democracia, 75, 77-110. https://www.redalyc.org/articulo.oa?id=357565037003

Pont Vidal, J. (2019b). Governança democrática. Para uma nova coordenação da sociedade. Tirant.

Pont Vidal, J. (coord.) (2018a). GIGAPP Estudios / Working papers (núms. 83-90, pp. 90-241, número especial, Observando la Gobernanza. Visiones desde la Amazonia). http://www.gigapp.org/ewp/index.php/GIGAPP-EWP/ issue/view/54.

Pont Vidal, J. (coord.) (2018b). Da governança hierárquica a interativa. Observações e análises. Paka-Tatu.

Pont Vidal, J. (2018c). Gobernanza. La coordinación entre democracia y jerarquía dinámica. Tirant.

Pont Vidal, J. (2017). Por meio da teoria. Enfoques neosistêmicos e pós-estruturalistas. Paka-Tatu.

Pressman, J. L., Wildavsky, A. (1973). Implementation: How Great Expectations in Washington are Dashed in Oakland; or, Why it's Amazing that Federal Programs Work at All. University of California Press.

Scharpf, F. y Mayntz, R. (1995). Gesellschaftliche Selbsregelung und politische Steuerung. Campus Verlag.

Sevilla, J. (coord.) (2010). La reforma de la Administración General del Estado. LID Empresarial.

Schimank, U. (2009). "Vater Staat»: ein vorhersehbares Comeback. Staatsverständnis und Staatstätigkeit in der Moderne. Der moderne Staat, 2(2), 249-270. https://www.budrich-journals.de/index.php/dms/article/ view/2879

Tait, A. y Richardson, K. (eds.) (2009). Complexity and Knowledge Management. Understanding the role of knowledge in the Management of the Social Networks. Information Age Publishing.

Teisman, G., Van Buuren, A. y Gerrits, L. (eds.) (2009). Managing Complex Governance Systems: Dynamics, Self Organization and Coevolution in Public Investments. Routledge.

Veit, S., Reichard, Ch. y Wewer, G. (2019). Handbuch zur Verwaltungsreform. Springer VS, Wiesbaden.

Willke, H. (1999). Systemtheorie II: Interventionstheorie. Einführung in die Theorie der Intervention in komplexe Sozialsysteme. UTB. 
GAPP. Nueva Época - N. ${ }^{\circ} 26$, julio 2021 - ISSN: 1989-8991 - DOI: https://doi.org/10.24965/gapp.i26.10798 - [Págs. 33-47]

Administración y Estado en el contexto post Covid-19: ¿Hacia un nuevo tipo de vínculo? Josep Pont Vidal

Willke, H. (1987). Differenzierung und Integration in Luhmanns Theorie sozialer Systeme, in: Hans Haferkamp und Michael Schmid (Hrsg.), Sinn, Kommunikation und soziale Differenzierung: Beiträge zu Luhmanns Theorie sozialer Systeme (pp. 247-274), Suhrkamp.

Willke, H. (1996). Dimensionen des Wissensmanagements: zum Zusammenhang von gesellschaftlicher und organisationaler Wissensbasierung. De Gruyter.

Willke, H. (1993). Systemtheorie entwickelter Gesellschaften. Dynamik und Riskanz moderner gesellschaftlicher Selbsorganization. Juventa.

Willke, H. (1992). Ironie des Staates: Grundlinien einer Staatstheorie polyzentrischer Gesellschaft. Suhrkamp. 